\title{
Straightening out rectangular differential inclusions
}

\author{
George J. Pappas, Shankar Sastry* \\ Department of Electrical Engineering and Computer Sciences, University of California at Berkeley, Berkeley, CA 94720, USA
}

Received 6 June 1997; received in revised form 10 February 1998; accepted 31 March 1998

\begin{abstract}
In this paper, the classic straightening out theorem from differential geometry is used to derive necessary and sufficient conditions for locally converting rectangular differential inclusions to constant rectangular differential inclusions. Both scalar and coupled differential inclusions are considered. The results presented in this paper have use in the area of computer aided verification of hybrid systems where they represent the frontier of the known decidable models of infinite state systems. (c) 1998 Elsevier Science B.V. All rights reserved.
\end{abstract}

Keywords: Differential inclusions; Hybrid systems; Coordinate transformation; Formal verification; Straightening out theorem

\section{Introduction}

In this paper, the problem of converting differential inclusions to constant differential inclusions is considered. The motivation for this work comes from the formal verification of hybrid systems. Hybrid systems are systems consisting of both discrete event and continuous dynamics. They arise in models of several distributed, multi-agent systems such as Intelligent Vehicle Highway Systems [14] and Air Traffic Management Systems [12]. The discrete event dynamics usually model high level decision making or synchronization among agents, while the continuous dynamics model low level control actions. The analysis, design and control of hybrid systems is a very important problem. Examples of techniques that have been proposed in the literature are [3-7, 9].

Computer aided verification is a formal approach for the analysis of hybrid systems. In the verification community, hybrid systems are modeled as hybrid automata where differential equations or inclusions exist in each discrete state of a finite state machine. Tran-

\footnotetext{
* Corresponding author. E-mail: sastry@eecs.berkeley.edu.
}

sition from one discrete state to another is triggered by guards on the variables of the system. An example of a hybrid automaton is shown in Fig. 1. Given a desired specification for a hybrid automaton, such as satisfying certain reachability properties, verification algorithms check whether the system indeed satisfies the desired specification by transforming the problem into a finite graph reachability or language containment problem. A very important issue in computer aided verification is the decidability and complexity of the resulting algorithms.

The state of the art in the verification of hybrid systems is that verification of initialized, rectangular hybrid automata is decidable [11]. Rectangular hybrid automata are automata where in each discrete location the continuous dynamics are described by decoupled, constant, rectangular differential inclusions. Thus, the time derivative of each variable must belong to a constant interval of the form $[a, b] \subset \mathbb{R}$, as shown in Fig. 1. Furthermore, checking properties on various relaxations of the above hybrid automaton model have been shown to be undecidable [8]. Therefore, initialized, rectangular hybrid automata are on the boundary between decidability and undecidability. 


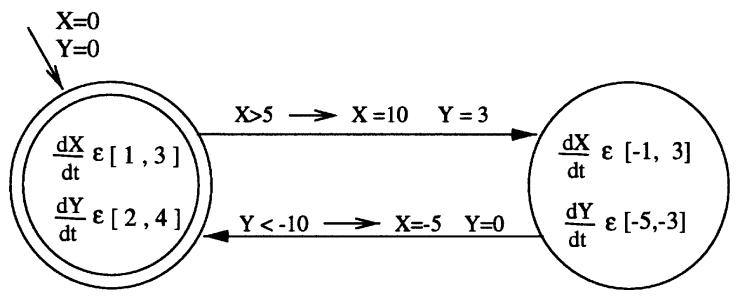

Fig. 1. Rectangular hybrid automaton.

It has also been recognized, mainly in the control community that is used to more sophisticated dynamical models, that the expressive power offered by a rectangular hybrid automaton is limited.

In an effort to expand the applicability of the decidability results just stated, we ask the natural question of when can we convert more general hybrid systems with more complicated dynamics in each discrete location to a hybrid system with decoupled, constant, differential inclusions? In addition, an answer to this question would also be useful as it could characterize the modeling frontier for the known decidability and undecidability results. Along this direction, in this paper we consider the following continuous version of this problem.

Problem 1 (Straightening out coupled, rectangular differential inclusions). Under what conditions can a coupled, rectangular differential inclusion of the form

$\dot{x}_{1} \in\left[f_{1}\left(x_{1}, \ldots, x_{n}\right), g_{1}\left(x_{1}, \ldots, x_{n}\right)\right]$

$\dot{x}_{n} \in\left[f_{n}\left(x_{1}, \ldots, x_{n}\right), g_{n}\left(x_{1}, \ldots, x_{n}\right)\right]$

where $x=\left[x_{1}, \ldots, x_{n}\right]^{\mathrm{T}} \in U \subseteq \mathbb{R}^{n}, f_{1}, \ldots, f_{n}, g_{1}, \ldots, g_{n}$ are smooth maps from $U$ to $\mathbb{R}$, and for each $1 \leqslant i \leqslant n$ and for all $x \in U, g_{i}(x)>f_{i}(x)$ be converted by a smooth coordinate change $z=\Phi(x)$ to a decoupled, constant, rectangular inclusion of the form

$\dot{z}_{1} \in\left[a_{1}, b_{1}\right]$

$\dot{z}_{n} \in\left[a_{n}, b_{n}\right]$

where $a_{i}, b_{i}$ are real constants for all $1 \leqslant i \leqslant n$ ?

In this endeavor, a generalized version of the straightening out (or flow box) theorem will be used in order to derive necessary and sufficient conditions for the solution of Problem 1. As a corollary, we will obtain conditions for converting decoupled, scalar rectangular inclusions. In Section 2, we review the necessary differential geometric tools and two versions of the straightening out theorem for differential equations. They will be used in Section 3, where necessary and sufficient conditions for the solvability of Problem 1 are derived. Finally, Section 4 contains conclusions.

\section{Straightening out differential equations}

Let $T_{x} \mathbb{R}^{n}$ denote the tangent space at $x \in \mathbb{R}^{n}$. Let $e_{i}=[0, \ldots, 1, \ldots, 0]^{\mathrm{T}}$ with 1 at the $i$ th position and $e_{1}, \ldots, e_{n}$ be the standard, orthonormal basis for $\mathbb{R}^{n}$. For smooth map $\Phi: \mathbb{R}^{n} \rightarrow \mathbb{R}^{m}$ with $x \in \mathbb{R}^{n}$ and $z=\Phi(x)$, we push forward tangent vectors from $T_{x} \mathbb{R}^{n}$ to $T_{z} \mathbb{R}^{m}$ using the induced push forward map $\Phi_{*}$ which is a linear map. A smooth vector field on $\mathbb{R}^{n}$ is a smooth map $f$ which places at each point $x \in \mathbb{R}^{n}$ a tangent vector $f(x) \in T_{x} \mathbb{R}^{n}$. Given a diffeomorphism $\Phi: \mathbb{R}^{n} \rightarrow \mathbb{R}^{n}$ and a smooth vector field $f$ on $\mathbb{R}^{n}$, we naturally define the vector field $\Phi_{*}(f)$ by pointwise assigning the tangent vector $\Phi_{*}(f(x))$ at $\Phi(x)$. The Lie bracket of two vector fields $f$ and $g$ on $\mathbb{R}^{n}$ is denoted by $[f, g]^{1}$ and given by

$[f, g]=\frac{\partial g}{\partial x} f-\frac{\partial f}{\partial x} g$.

The Lie bracket commutes with the push forward map $\Phi_{*}$ of a diffeomorphism $\Phi$, thus

$$
\Phi_{*}([f, g])=\left[\Phi_{*}(f), \Phi_{*}(g)\right] .
$$

We now present without proof the $\mathbb{R}^{n}$ version of the

Theorem 1 (Flow box or straightening out theorem). Let $f$ be a smooth vector field on $\mathbb{R}^{n}$ with $f\left(x_{0}\right) \neq 0$ at some $x_{0} \in \mathbb{R}^{n}$. Then there exists a neighborhood $U$ of $x_{0}$ and a change of coordinates $z=\Phi(x)$ such that for all $z \in \Phi(U), \Phi_{*}(f)$ is expressed as

$$
\Phi_{*}(f)=e_{1} .
$$

Thus, given a differential equation of the form

$\dot{x}=f(x)$,

where $x \in \mathbb{R}^{n}$ and $f: \mathbb{R}^{n} \rightarrow \mathbb{R}^{n}$ is smooth, then away from equilibria, $f(x) \neq 0$, there exists a local change

\footnotetext{
${ }^{1}$ Note that $[\cdot, \cdot]$ is used to denote both Lie brackets as well as intervals. The usage will be clear from the context.
} 
of coordinates $z=\Phi(x)$ such that in the $z$ coordinates the differential equation is expressed as

$\dot{z}_{1}=1, \dot{z}_{2}=0, \ldots, \dot{z}_{n}=0$.

Note that the straightening out theorem is a local and non-constructive result. Obtaining the desired diffeomorphism $\Phi$ usually involves explicit integration of the system since the change of coordinates is simply the time parameterization of the integral curves $\left(z_{1}\right)$ along with the leaves of the resulting foliation $\left(z_{2}, \ldots, z_{n}\right)$ which is induced by integrating the system. Theorem 1 is already interesting in that it allows transforming differential equations away from equilibria to equations with constant rates or clocks. The flow box theorem could therefore be used in converting hybrid systems with the same differential equation in each discrete location to timed automata, which is a decidable class of systems [2]. In the case where different vector fields are present in different discrete states, the following theorem which can be considered a generalization of Theorem 1 for multiple vector fields is useful.

Theorem 2 (Straightening out multiple vector fields). Let $f_{1}, \ldots, f_{k}$ be $k$ smooth, linearly independent vector fields in a neighborhood of $x_{0} \in \mathbb{R}^{n}$ satisfying

$\left[f_{i}, f_{j}\right]=0, \quad 1 \leqslant i, j \leqslant k$.

Then there exists a change of coordinates $z=\Phi(x)$ and a neighborhood $U$ around $x_{0} \in \mathbb{R}^{n}$, such that

$\Phi_{*}\left(f_{i}\right)=e_{i}$

for all $1 \leqslant i \leqslant k$ and for all $z \in \Phi(U)$.

Therefore given $n$ differential equations of the form $\dot{x}=f_{i}(x)$,

where $1 \leqslant i \leqslant n, x \in \mathbb{R}^{n}$ and $f_{i}: \mathbb{R}^{n} \rightarrow \mathbb{R}^{n}$ are smooth, then at any $x_{0} \in \mathbb{R}^{n}$ where the vectors $\left\{f_{i}\left(x_{0}\right)\right\}_{i=1}^{n}$ is a linearly independent $\operatorname{set}^{2}$ and the Lie bracket conditions hold, there exists a local change of coordinates $z=\Phi(x)$ such that in the $z$ coordinates the $i$ th differential equation is expressed as

$\dot{z}_{1}=0, \ldots, \dot{z}_{i}=1, \ldots, \dot{z}_{n}=0$.

Like the Flow Box Theorem, Theorem 2 is also local and non-constructive. The Lie bracket condition,

\footnotetext{
${ }^{2}$ Note that linear independence at $x_{0}$ requires that $x_{0}$ is not an equilibrium of any of the $n$ vector fields. By smoothness, the linear independence condition extends to a neighborhood of $x_{0}$.
}

which simply says that the flows of the vector fields commute, is necessary in order for the change of coordinates to be well defined. More important though, in the new coordinates, the vector fields in addition to being straightened out are also decoupled.

A much more detailed exposition of the above material may be found in numerous differential geometry books such as $[13,1]$.

\section{Straightening out differential inclusions}

In general, a differential inclusion on $\mathbb{R}^{n}$ is defined as

$\dot{x} \in F(x)$,

where $F$ is a map which at each $x \in \mathbb{R}^{n}$ assigns a subset of $T_{x} \mathbb{R}^{n}$. Given a smooth change of coordinates $\Phi: \mathbb{R}^{n} \rightarrow \mathbb{R}^{n}$ and differential inclusion (8), we can naturally push forward the differential inclusion by pointwise assigning to each $z=\Phi(x)$ the push forward of all tangent vectors belonging in $F(x)$. Thus

$\dot{z} \in \Phi_{*}(F(x))$

is the differential inclusion resulting from the change of coordinates. In this paper we focus on rectangular differential inclusions of the form

$\dot{x}_{1} \in\left[f_{1}\left(x_{1}, \ldots, x_{n}\right), g_{1}\left(x_{1}, \ldots, x_{n}\right)\right]$

$\dot{x}_{n} \in\left[f_{n}\left(x_{1}, \ldots, x_{n}\right), g_{n}\left(x_{1}, \ldots, x_{n}\right)\right]$

where the derivative of each coordinate lies in an interval. We are now ready to proceed with the main theorem.

Theorem 3 (Necessary and sufficient conditions for coupled rectangular inclusions). Consider the coupled, rectangular differential inclusion in $\mathbb{R}^{n}$,

$\dot{x}_{1} \in\left[f_{1}\left(x_{1}, \ldots, x_{n}\right), g_{1}\left(x_{1}, \ldots, x_{n}\right)\right]$

$\dot{x}_{n} \in\left[f_{n}\left(x_{1}, \ldots, x_{n}\right), g_{n}\left(x_{1}, \ldots, x_{n}\right)\right]$

where $x=\left[x_{1}, \ldots, x_{n}\right]^{\mathrm{T}} \in U \subseteq \mathbb{R}^{n}, f_{1}, \ldots, f_{n}, g_{1}, \ldots, g_{n}$ are smooth maps from $U$ to $\mathbb{R}$, and for each $i$ and for all $x \in U$ we have $g_{i}(x)>f_{i}(x)$. Then there exists a local change of coordinates $z=\Phi(x)$ on $U$ such 
that in the new coordinates the differential inclusion is expressed as

$\dot{z}_{1} \in\left[a_{1}, b_{1}\right]$

$\dot{z}_{n} \in\left[a_{n}, b_{n}\right]$

if and only if for all $x \in U$ and for all $1 \leqslant i, j \leqslant n$,

$\left[f_{i}(x) e_{i}, g_{j}(x) e_{j}\right]=0$,

$\left[f_{i}(x) e_{i}, f_{j}(x) e_{j}\right]=0$,

and for all $1 \leqslant i \leqslant n$ and for all $x \in U$ there exist $k_{i} \in \mathbb{R}$, such that either

$g_{i}(x)=k_{i} f_{i}(x) \quad$ or $\quad f_{i}(x)=k_{i} g_{i}(x)$.

Proof. Before we begin with the proof, we remark that conditions (13)-(15) contain some redundancy. However, a minimal set of conditions would be notationally complicated.

(Necessity) A more convenient representation of the rectangular inclusion (10) is given by the following expression

$$
\begin{aligned}
\dot{x} & =\left[\begin{array}{c}
\dot{x}_{1} \\
\vdots \\
\dot{x}_{n}
\end{array}\right] \in F(x) \\
& =F_{1}(x)+F_{2}(x)+\cdots+F_{n}(x),
\end{aligned}
$$

$$
\begin{aligned}
F_{i}(x) & =\operatorname{co}\left\{\left[\begin{array}{c}
0 \\
\vdots \\
f_{i}(x) \\
\vdots \\
0
\end{array}\right],\left[\begin{array}{c}
0 \\
\vdots \\
g_{i}(x) \\
\vdots \\
0
\end{array}\right]\right\} \\
& =\operatorname{co}\left\{f_{i}(x) e_{i}, g_{i}(x) e_{i}\right\}
\end{aligned}
$$

where $\operatorname{co}\left\{p_{1}, p_{2}\right\}$ stands for the convex hull of vectors $p_{1}$ and $p_{2}$. Note that for $i \neq j$, any vector in $F_{i}(x)$ is linearly independent from any vector in $F_{j}(x)$. Performing the change of coordinates $z=\Phi(x)$ results in

$$
\begin{aligned}
\dot{z} & \in \Phi_{*}(F(x)) \\
& =\Phi_{*}\left(F_{1}(x)+F_{2}(x)+\cdots+F_{n}(x)\right) .
\end{aligned}
$$

By the linearity of $\Phi_{*}$ we have that

$\dot{z} \in \Phi_{*}\left(F_{1}(x)\right)+\Phi_{*}\left(F_{2}(x)\right)+\cdots+\Phi_{*}\left(F_{n}(x)\right)$.
Since $\Phi_{*}$ is pointwise an isomorphism, we retain the property that any vector from $\Phi_{*}\left(F_{i}(x)\right)$ is linearly independent from any vector in $\Phi_{*}\left(F_{j}(x)\right)$ for $i \neq j$.

Now, by assumption, the change of coordinates results in inclusion (12) which is also expressed as

$\dot{z}=\left[\begin{array}{c}\dot{z}_{1} \\ \vdots \\ \dot{z}_{n}\end{array}\right] \in Z=Z_{1}+Z_{2}+\cdots+Z_{n}$,

where $Z_{i}$ is the constant interval

$Z_{i}=\operatorname{co}\left\{\left[\begin{array}{c}0 \\ \vdots \\ a_{i} \\ \vdots \\ 0\end{array}\right],\left[\begin{array}{c}0 \\ \vdots \\ b_{i} \\ \vdots \\ 0\end{array}\right]\right\}=\operatorname{co}\left\{a_{i} e_{i}, b_{i} e_{i}\right\}$.

Note again that for $i \neq j$, any vector from $Z_{i}$ is linearly independent from any vector in $Z_{j}$. By assumption we then have that

$$
\begin{aligned}
\dot{z} & \in \Phi_{*}\left(F_{1}(x)\right)+\Phi_{*}\left(F_{2}(x)\right)+\cdots+\Phi_{*}\left(F_{n}(x)\right) \\
& =Z_{1}+Z_{2}+\cdots+Z_{n} .
\end{aligned}
$$

Since for all $i \neq j$, vectors in $\Phi_{*}\left(F_{i}(x)\right)$ (also $Z_{i}$ ) are linearly independent from vectors in $\Phi_{*}\left(F_{j}(x)\right)$ (respectively $Z_{j}$ ) then Eq. (22) requires that for each $i$ there exists some $j_{i}$ such that $\Phi_{*}\left(F_{i}(x)\right)=Z_{j_{i}}$. Therefore, up to a permutation of the indices, the sets $\Phi_{*}\left(F_{i}(x)\right)$ are equal to the sets $Z_{i}$.

In general, for linear map $A$ and vectors $p_{1}, p_{2}$ the following property of convex hulls

$A \operatorname{co}\left\{p_{1}, p_{2}\right\}=\operatorname{co}\left\{A p_{1}, A p_{2}\right\}$

can be easily checked. By applying this property on Eqs. (19) and (17) we obtain that

$$
\begin{aligned}
\Phi_{*}\left(F_{i}(x)\right) & =\Phi_{*}\left(\operatorname{co}\left\{f_{i}(x) e_{i}, g_{i}(x) e_{i}\right\}\right) \\
& =\operatorname{co}\left\{\Phi_{*}\left(f_{i}(x) e_{i}\right), \Phi_{*}\left(g_{i}(x) e_{i}\right)\right\} .
\end{aligned}
$$

The above calculations essentially show that in order to push forward a rectangular differential inclusion, one only needs to push forward the finite number of vector fields that are needed to define the rectangular set of tangent vectors.

But since $\Phi_{*}\left(F_{i}(x)\right)=Z_{j_{i}}$, condition (24) results in $\operatorname{co}\left\{\Phi_{*}\left(f_{i}(x) e_{i}\right), \Phi_{*}\left(g_{i}(x) e_{i}\right)\right\}=\operatorname{co}\left\{a_{j_{i}} e_{j_{i}}, b_{j_{i}} e_{j_{i}}\right\}$

which means that either

$\Phi_{*}\left(f_{i}(x) e_{i}\right)=a_{j_{i}} e_{j_{i}} \quad$ and $\quad \Phi_{*}\left(g_{i}(x) e_{i}\right)=b_{j_{i}} e_{j_{i}}$ 


$$
\Phi_{*}\left(f_{i}(x) e_{i}\right)=b_{j_{i}} e_{j_{i}} \quad \text { and } \quad \Phi_{*}\left(g_{i}(x) e_{i}\right)=a_{j_{i}} e_{j_{i}} .
$$

Assume without loss of generality that the first case holds (Eq. 26). Then for all $0 \leqslant i, l \leqslant n$,

$$
\begin{aligned}
\Phi_{*}\left(\left[f_{i}(x) e_{i}, g_{l}(x) e_{l}\right]\right) & =\left[\Phi_{*}\left(f_{i}(x) e_{i}\right), \Phi_{*}\left(g_{l}(x) e_{l}\right)\right] \\
& =\left[a_{j_{i}} e_{j_{i}}, b_{j_{l}} e_{j_{l}}\right]=0
\end{aligned}
$$

which results in the necessary conditions

$\left[f_{i}(x) e_{i}, g_{l}(x) e_{l}\right]=0 \quad$ for all $0 \leqslant i, l \leqslant n$

since $\Phi_{*}$ is pointwise an isomorphism. In a similar manner one obtains

$\left[f_{i}(x) e_{i}, f_{l}(x) e_{l}\right]=0 \quad$ for all $0 \leqslant i, l \leqslant n$.

In addition, since $g_{i}(x)>f_{i}(x)$, if $f_{i}(x) \neq 0$ we can express $g_{i}(x)$ as a nonlinear function of $f_{i}(x)$ by $g_{i}(x)=k_{i}(x) f_{i}(x)$ (if $f_{i}(x)=0$ then express $f_{i}(x)$ as $g_{j}(x)$ multiplied by zero and proceed in the same way). Then

$$
\begin{aligned}
b_{j_{i}} e_{j_{i}} & =\Phi_{*}\left(g_{i}(x) e_{i}\right)=\Phi_{*}\left(k_{i}(x) f_{i}(x) e_{i}\right) \\
& =k_{i}(x) \Phi_{*}\left(f_{i}(x) e_{i}\right)=k_{i}(x) a_{j_{i}} e_{j_{i}}
\end{aligned}
$$

must hold for all $x \in U$. Thus $k_{i}(x)$ must be constant and $g_{i}(x)$ must be a constant multiple of $f_{i}(x)$ for all $x \in U$. Note that for each $i$ either $f_{i}(x)$ or $g_{i}(x)$ can be zero (but not both since $g_{i}(x)>f_{i}(x)$ ). However, if $f_{i}(x)$ or $g_{i}(x)$ is zero at some point $x_{0}$, say $g_{i}\left(x_{0}\right)=0$ and $f_{i}\left(x_{0}\right) \neq 0$, then smoothness and the fact that $g_{i}(x)$ must be a constant multiple of $f_{i}(x)$ for all $x \in U$, force $g_{i}(x)$ to be identically zero on $U$.

(Sufficiency) Consider conditions (13)-(15) and assume without loss of generality that for all $i$ $f_{i}(x) \neq 0$ (if $f_{i_{0}}=0$ for some $i_{0}$ then pick $g_{i_{0}}$ which must be nonzero and proceed in a similar way). Then, the set of vector fields

$\left\{f_{i}(x) e_{i}\right\}_{i=1}^{n}$

satisfies the conditions of Theorem 2 . Thus, there exists a diffeomorphism $z=\Phi(x)$ such that

$\Phi_{*}\left(f_{i}(x) e_{i}\right)=e_{i}$.

Now pushing forward the rectangular inclusion

$\dot{x} \in F_{1}(x)+F_{2}(x)+\cdots+F_{n}(x)$ by $\Phi_{*}$ results in

$$
\begin{aligned}
\dot{z} & \in \Phi_{*}(F(x)) \\
& =\Phi_{*}\left(F_{1}(x)+F_{2}(x)+\cdots+F_{n}(x)\right) \\
& =\Phi_{*}\left(F_{1}(x)\right)+\Phi_{*}\left(F_{2}(x)\right)+\cdots+\Phi_{*}\left(F_{n}(x)\right) .
\end{aligned}
$$

But since for each $i$ and for all $x$ we have $g_{i}(x)=k_{i} f_{i}(x)$ for some constant $k_{i}$ (positive, negative or zero), we obtain

$$
\begin{aligned}
\Phi_{*}\left(F_{i}(x)\right) & =\Phi_{*}\left(\operatorname{co}\left\{f_{i}(x) e_{i}, k_{i} f_{i}(x) e_{i}\right\}\right) \\
& =\operatorname{co}\left\{\Phi_{*}\left(f_{i}(x) e_{i}\right), \Phi_{*}\left(k_{i} f_{i}(x) e_{i}\right)\right\} \\
& =\operatorname{co}\left\{e_{i}, k_{i} e_{i}\right\}
\end{aligned}
$$

and thus in the $z$ coordinates we obtain the inclusion

$$
\begin{gathered}
\dot{z}_{1} \in\left[1, k_{1}\right] \\
\vdots \\
\dot{z}_{n} \in\left[1, k_{n}\right]
\end{gathered}
$$

Note that some of the $k_{i}$ may be zero or even negative in which case the corresponding intervals must be flipped. This completes the proof.

Note that the proof of Theorem 3 depends on the fact that $g_{i}(x)>f_{i}(x)$ for all $i$. It is therefore not a generalization of the straightening out theorem for differential equations.

Even though straightening out a differential equation is always possible away from an equilibrium, straightening out a rectangular differential inclusion, requires straightening out many vector fields, while using the same change of coordinates. This places restrictions on the types of rectangular differential inclusions that can be straightened out. The following corollary shows how restrictive this class is.

Example. Consider the coupled differential inclusion

$\dot{x}_{1} \in\left[f_{1}\left(x_{1}, x_{2}\right), g_{1}\left(x_{1}, x_{2}\right)\right]$,

$\dot{x}_{2} \in\left[f_{2}\left(x_{1}, x_{2}\right), g_{2}\left(x_{1}, x_{2}\right)\right]$,

where we have $f_{1}\left(x_{1}, x_{2}\right) \neq 0$ and $f_{2}\left(x_{1}, x_{2}\right) \neq 0$ on some set $U \subseteq \mathbb{R}^{2}$. Then conditions (15) require that $g_{i}\left(x_{1}, x_{2}\right)$ is a constant multiple of $f_{i}\left(x_{1}, x_{2}\right)$. Thus necessary conditions $(13,14)$ reduce to simply checking whether

$\left[f_{1}\left(x_{1}, x_{2}\right) e_{1}, f_{2}\left(x_{1}, x_{2}\right) e_{2}\right]=0$ 
as all other Lie brackets are guaranteed to be zero if the above one is. But

$$
\begin{aligned}
& {\left[f_{1}\left(x_{1}, x_{2}\right) e_{1}, f_{2}\left(x_{1}, x_{2}\right) e_{2}\right]=0} \\
& \Rightarrow\left[\begin{array}{l}
\frac{\partial f_{1}}{\partial x_{2}} f_{2} \\
\frac{\partial f_{2}}{\partial x_{1}} f_{1}
\end{array}\right]=0 .
\end{aligned}
$$

But since $f_{1} \neq 0$ and $f_{2} \neq 0$ on $U$, this requires

$$
\frac{\partial f_{1}}{\partial x_{2}}=0 \quad \frac{\partial f_{2}}{\partial x_{1}}=0
$$

which means that it is necessary for the rectangular inclusion to be already decoupled!

The above example suggests that the conditions of Theorem 3 are quite restrictive. In the case that $f_{i}(x)$ and $g_{i}(x)$ depend on $x_{i}$ alone, the Lie bracket conditions (13), (14) are trivially satisfied. As a corollary of Theorem 3, we obtain the following straightening out theorem for decoupled, rectangular inclusions.

Corollary 1 (Straightening out decoupled differential Inclusions). Consider the scalar differential inclusion

$\dot{x} \in[f(x), g(x)]$

with $x \in U \subseteq \mathbb{R}, f, g: U \rightarrow \mathbb{R}$ smooth, and assume that for all $x \in U$ we have $g(x)>f(x)$. Then there exists a local change of coordinates $z=\Phi(x)$ such that in the new coordinates the differential inclusion is expressed as

$\dot{z} \in[a, b]$

if and only if for all $x \in U$ either $g(x)$ is a constant multiple of $f(x) \neq 0$ or $f(x)$ is a constant multiple of $g(x) \neq 0$.

As a corollary of Corollary 1 we obtain

Corollary 2. The following scalar inclusions can be locally transformed to constant rectangular differential inclusions:

- Linear differential inclusions: $\dot{x} \in[a, b] x, x \neq 0$.

- Nonlinear differential inclusions: $\dot{x} \in[0, f(x)]$, $f(x)>0$.

- Nonlinear differential inclusions: $\dot{x} \in[f(x), 0]$, $f(x)<0$.

- Nonlinear differential inclusions: $\dot{x} \in[a, b] f(x)$, $f(x) \neq 0$.

Corollaries 1 and 2 show that scalar rectangular differential inclusions cannot be straightened out unless one boundary vector field, $g(x)$, is a constant multiple of the other, $f(x)$. This result is intuitively clear. By Theorem 1, any vector field, say $f(x)$, can be straightened out away from singularities. But if the same diffeomorphism must also straighten the flows of the other vector field, $g(x)$, then $g(x)$ must be a constant multiple of $f(x)$. But if $g(x)$ is a constant multiple of $f(x)$, then after factorization, we obtain that a differential inclusion of the form $\dot{x} \in[a, b] f(x)$ is the limiting case of an inclusion which can be straightened out.

Example. Consider the following simple linear differential inclusion in $U=\{x \in \mathbb{R} \mid x>0\}$,

$\dot{x} \in[3,5] x$

Note that $z=\ln x$ satisfies

$\dot{z}=\frac{\partial \ln x}{\partial x} \dot{x} \in \frac{1}{x}[3,5] x=[3,5]$

and the inclusion is straightened out on $U$.

\section{Conclusions}

In this paper, the problem of straightening out rectangular differential inclusions was considered. The results presented in this paper could be used to potentially expand the domain of the decidability results in the area of formal verification of hybrid systems. However, given the restrictive nature of the necessary conditions, they present a serious barrier extending the decidable class of hybrid systems. In an effort to computationally analyze more complicated hybrid systems, one has to overapproximate arbitrary rectangular inclusions by rectangular inclusions which satisfy the necessary and sufficient conditions derived in this paper. This is the notion of system abstractions [10] which is an area for further research.

\section{Acknowledgements}

This work was supported by the Army Research Office under grants DAAH 04-95-1-0588 and DAAH 04-96-1-0341.

\section{References}

[1] R. Abraham, J. Marsden, T. Ratiu, Manifolds, Tensor Analysis and Applications, Applied Mathematical Sciences, 2nd ed., Springer, New York, 1988. 
[2] R. Alur, D.L. Dill, A theory of timed automata, Theoret. Comput. Sci. 126 (1994) 183-235.

[3] R. Alur, T.A. Henzinger, E.D. Sontag (Eds.), Hybrid Systems III, Lecture Notes in Computer Science, vol. 1066, Springer, Berlin, 1996.

[4] P. Antsaklis, W. Kohn, A. Nerode, S. Sastry (Eds.), Hybrid Systems II, Lecture Notes in Computer Science, vol. 999, Springer, Berlin, 1995.

[5] P. Antsaklis, W. Kohn, A. Nerode, S. Sastry (Eds.), Hybrid Systems IV, Lecture Notes in Computer Science, vol. 1273, Springer, Berlin, 1997.

[6] R.L. Grossman, A. Nerode, A.P. Ravn, H. Rischel (Eds.), Hybrid Systems, Lecture Notes in Computer Science, vol. 736, Springer, Berlin, 1993.

[7] T. Henzinger, S. Sastry (Eds.), Hybrid Systems: Computation and Control, Lecture Notes in Computer Science, vol. 1386, Springer, Berlin, 1998.

[8] T.A. Henzinger, P.W. Kopke, A. Puri, P. Varaiya, What's decidable about hybrid automata? in: Proc. 27th Annual Symp. on Theory of Computing, ACM Press, New York, 1995, pp. 373-382.
[9] O. Maler (Ed.), Hybrid and Real Time Systems, Lecture Notes in Computer Science, vol. 1201, Springer, Berlin, 1997.

[10] G.J. Pappas, S. Sastry, Towards continuous abstractions of dynamical and control systems, in: P. Antsaklis, W. Kohn, A. Nerode, S. Sastry (Eds.), Hybrid Systems IV, Lecture Notes in Computer Science, vol. 1273, Springer, Berlin, 1997, pp. 329-341.

[11] A. Puri, P. Varaiya, Decidability of hybrid systems with rectangular differential inclusions, in: Computer Aided Verification, Lecture Notes in Computer Science, vol. 818, Springer, Berlin, 1994, pp. 95-104.

[12] S. Sastry, G. Meyer, C. Tomlin, J. Lygeros, D. Godbole, G. Pappas, Hybrid control in air traffic management systems, in: Proc. 1995 IEEE Conf. in Decision and Control, New Orleans, LA, December 1995, pp. 1478-1483.

[13] M. Spivak, A Comprehensive Introduction to Differential Geometry, 2nd ed., Publish or Perish, Houston, TX, 1979.

[14] P. Varaiya, Smart cars on smart roads: problems of control, IEEE Trans. Automat. Control AC-38 (2) (1993) 195-207. 